\title{
Modelling elastomer buffers with DyMoRail
}

\author{
Elisabeth Dumont Werner Maurer \\ Institut für Angewandte Mathematik und Physik \\ Zürcher Hochschule für Angewandte Wissenschaften \\ Technikumstrasse 9, Winterthur, 8401 Switzerland
}

\begin{abstract}
In this paper a model for elastomer buffers for longitudinal railway vehicle dynamics is presented. This model is part of the more extended DyMoRail library which allows to simulate longitudinal dynamics of entire railway trains. With this library an efficient simulation of complete train compositions in various combinations is possible. The elastomer buffer can be used in combination with other buffer models and couplers in different test scenarios. We present details of our rubber spring model based on the one-dimensional, non-linear rubber spring model proposed by M. Berg [1][2][3]. To illustrate the behavior of the friction force modelled in the latter, it is compared to a diode model for Coulomb friction similar to the one in the Modelica Standard Library. Simulations for 40 J-buffer known as "Miner40" used for freight waggons during shunting at speeds up to $12 \mathrm{~km} / \mathrm{h}$ are shown. Also shown are simulations of an entire S-Bahn combination with sixteen cars and fifteen elastomer buffers.
\end{abstract}

Keywords: mechanics, railway

\section{Introduction}

The tough competition in the railway industry is forcing operators to continuously set higher quality and comfort standards. Buffers on railway vehicles, as fabricated by Schwab Verkehrstechnik, are no more simple devices but have to be considered Hi-Tech components. They have to be be absolutely reliable and present optimal properties to absorb run-up energy from trailing wagons safely. They have to absorb minor impacts, take up slack between locomotive and wagons and bear the load of preceding wagons when pushing. Years ago it was good enough for couplers and buffers to fulfill UIC (International Union of Railways) stan- dards. But nowadays manufacturers only survive in this competitive market if they are able to offer optimized solutions regarding force, energy absorption, and driving comfort. The manufacturer has to be able to react quickly and flexibly to the challenges of rail operators and rolling stock manufacturers and offer final products and customized solutions with high customer value landlord. Since hardware tests are extremely costly, modeling and simulation step into the optimization process. DyMoRail allows to optimize buffers and couplers in different configurations for safety, energy absorption and costs. Train composition can be chosen by the user.

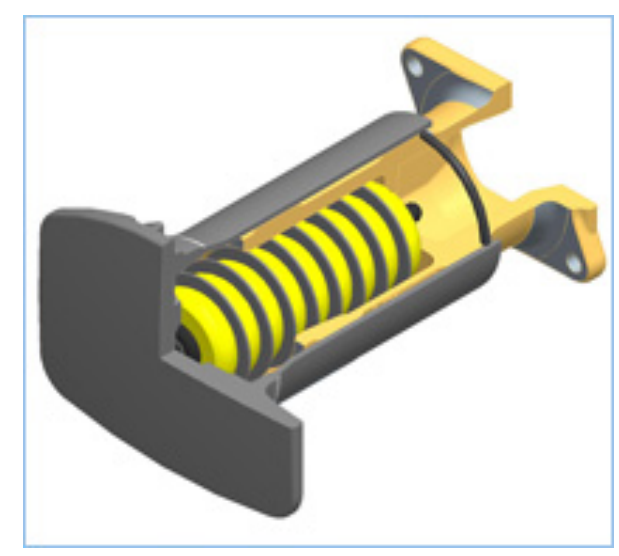

Figure 1: Drawing of a buffer (Seitenpuffer Kategorie A by Schwab Verkehrstechnik).

Schwab Verkehrstechnik AG and ZHAW carried out a project funded by CTI (Swiss Federal Commission for Technology and Innovation) to develop a tool which allows to simulate longitudinal dynamics of entire railway trains. During the following years a Modelica library has been developed, which is called DyMoRail. The DyMoRail library allows an efficient simulation of complete train compositions in various configurations. The library has been presented at Modelica 2012 [4] and a description of the coupler models can be 
found in references [5][6]. In this paper we focus on our elastomer buffer library and we will present details of our rubber spring model.

The elastomer buffers consists of a series of ring rubber pads put in series separated by metallic shims, as shown in Figure 1. When compressed energy is stored within the material of the rubber pads as strain energy. The energy is dissipated within the material during both the compression and extension of the material due to the internal friction rising from the long cross linked polymers within the material. Elastomer buffers present a non linear force-stroke-diagram.

\section{Library}

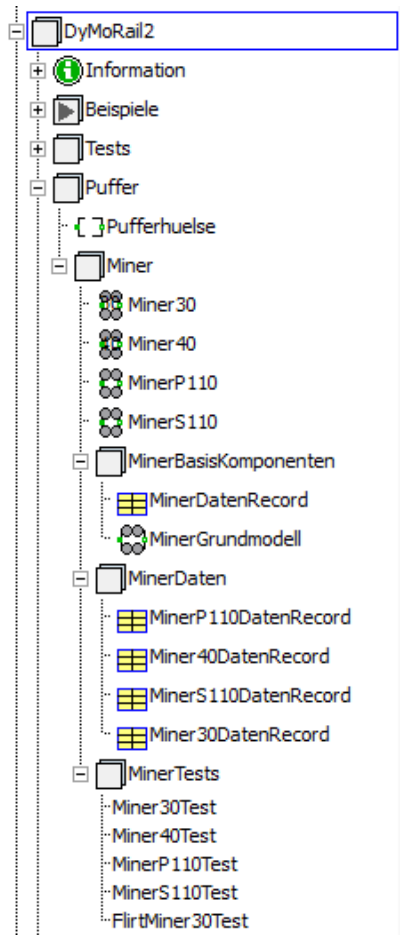

Figure 2: Structure of the buffer sublibrary "Puffer".

The buffer library, shown in Figure 2 has been completely redesigned. All buffer models are based on the partial model "Pufferhuelse" (buffer bush) where the different modes and overall buffer force are modeled. A detailed description of the connectors and the partial model used to build the buffer model is shown in Figure 3. Force and stroke are calculated and, in addition, the energy absorbed by the buffer. The modes are divided into two categories: four operation modes and three motion modes. For the operation modes, we distinguish

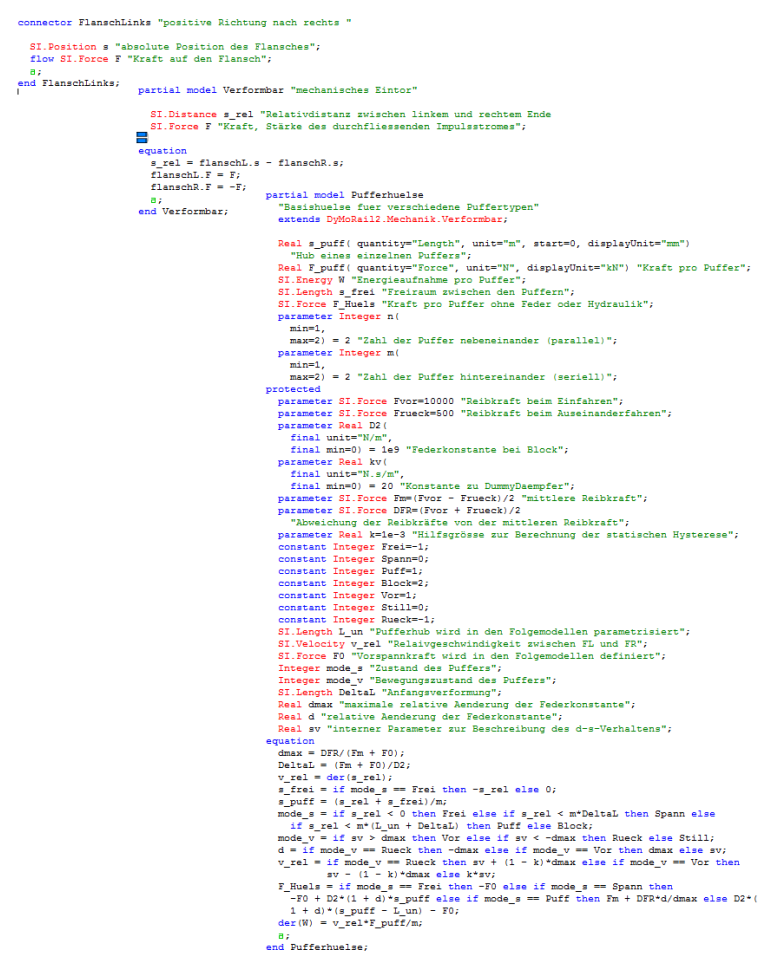

Figure 3: Models for connectors and partial models.

free, pretension, deformation and arrested. In free mode, the buffer plates do not touch and the force is zero. In the pretension mode the force increases slowly. In the deformation mode the buffer spring is loaded. In the arrested mode the force increases steeply. The motion modes include forward, backward and halt. They describe the current motion of the bush. We will come back to these modes, when discussing the friction model. In the basic buffer model, the Coulomb friction force in the buffer bush is also modeled. A maximum of two buffers can be placed both in series and parallel. European standard configuration consists of two buffers in parallel. A crash between two cars is modelled as two buffers in series on each side, one buffer per car.

Based on this partial model, the actual buffer model is built, including the elastomer spring. Data sheets provided by the manufacturer are included with the help of records in "Miner Daten".

For their acceptance test, railway companies define several crash scenarios with different crash partners at different speeds. Those test scenarios are implemented by means of a test library "Miner Tests". It allows for comparability, consistency and easy reproduction of the total set of test cases. 


\section{Model}

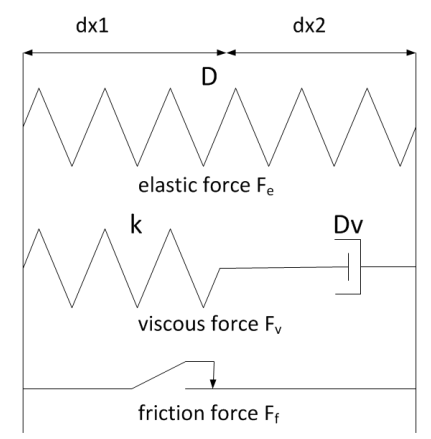

Figure 4: Model for a rubber spring proposed by M. Berg.

For model implementation of the DyMoRail library, the Modelica based simulation software Dymola is used. The elastomer model is taken from the non-linear rubber spring model by $\mathrm{M}$. Berg[1][2][3]. The model proposed in these papers is one-dimensional and based on the superposition of three forces (elastic, friction and viscous force) and contains five parameters. For better understanding, a schematic drawing is shown in Figure 4.

In the model by M. Berg, the elastic force is linearly modelled with a stiffness constant $\mathrm{D}$ :

$$
F_{e}=D \cdot x
$$

The viscous force is modelled by a linear spring (spring constant Dv) in series with a linear viscous damper (damping constant $\mathrm{k}$ ):

$$
F_{v}=D v \cdot x-k v
$$

The friction force $F_{\text {Coul }}$ depends both on the displacement $x$ and on a reference state $\left(x_{s}, F_{R s}\right)$ in the friction force versus displacement characteristic. Depending on the position relative to this reference state, the friction force is expressed with two parameters maximum force $F_{R \max }$ and constant $x_{2}$. A small value of $x_{2}$ gives a steep increase in the friction force and thereby high frictional stiffness. The friction force $F_{\text {Coul }}$ in the model is, depending on how $x$ is related to the reference displacement $x_{s}$, defined by the equations below.

The reference state is set to $x_{s}=0$ and $F_{R s}=0$.

$$
F_{\text {Coul }}=F_{\text {Rmax }} \frac{s_{\text {rel }}}{s_{\text {rel }}+x_{2}}
$$

For backward movement $x s=x 1$ and $F R s=$ FR1:

$$
\begin{aligned}
& F_{\text {Coul }}=F_{R 1}+\left(F_{R \max }+F_{R 1}\right) \\
& \times \frac{s_{\text {rel }}-x_{1}}{x_{2}\left(1+\frac{F_{R 1}}{F_{R \max }}\right)-\left(s_{\text {rel }}-x_{1}\right)}
\end{aligned}
$$

For backward movement $x s=x 1$ and $F R s=$ $F R 1=F R \max$ :

$$
F_{\text {Coul }}=F_{\text {Rmax }}\left(1-2 \frac{s_{\text {rel }}-x_{1}}{2 x_{2}-\left(s_{\text {rel }}-x_{1}\right)}\right)
$$

For forward movement $x s=x 3$ and $F R s=$ FR3:

$$
\begin{aligned}
& F_{\text {Coul }}=F_{R 3}+\left(F_{R \max }-F_{R 3}\right) \\
& \times \frac{s_{\text {rel }}-x_{3}}{x_{2}\left(1-\frac{F_{R 3}}{F_{R \max }}\right)+\left(s_{\text {rel }}-x_{3}\right)}
\end{aligned}
$$

For forward movement $x s=x 3$ and $F R s=$ $F R 3=-F R \max$ :

$$
F_{\text {Coul }}=F_{\text {Rmax }}\left(s \frac{s_{\text {rel }}-x_{3}}{2 x_{2}+\left(s_{\text {rel }}-x_{3}\right)}-1\right)
$$

When the buffer is at rest $x s=x 5$ and $F R s=$ FR5:

$$
F_{\text {Coul }}=F_{R 5}+F_{R \max } \frac{s_{r e l}-x_{5}}{x_{2}+\left(s_{r e l}-x_{5}\right)}
$$

When the buffer is at rest mode $x s=x 5$ and $F R s=F R 5= \pm F R \max :$

$$
F_{\text {Coul }}=F_{\text {Rmax }}\left( \pm 1+\frac{s_{\text {rel }}-x_{5}}{x_{2}+\left(s_{\text {rel }}-x_{5}\right)}\right)
$$

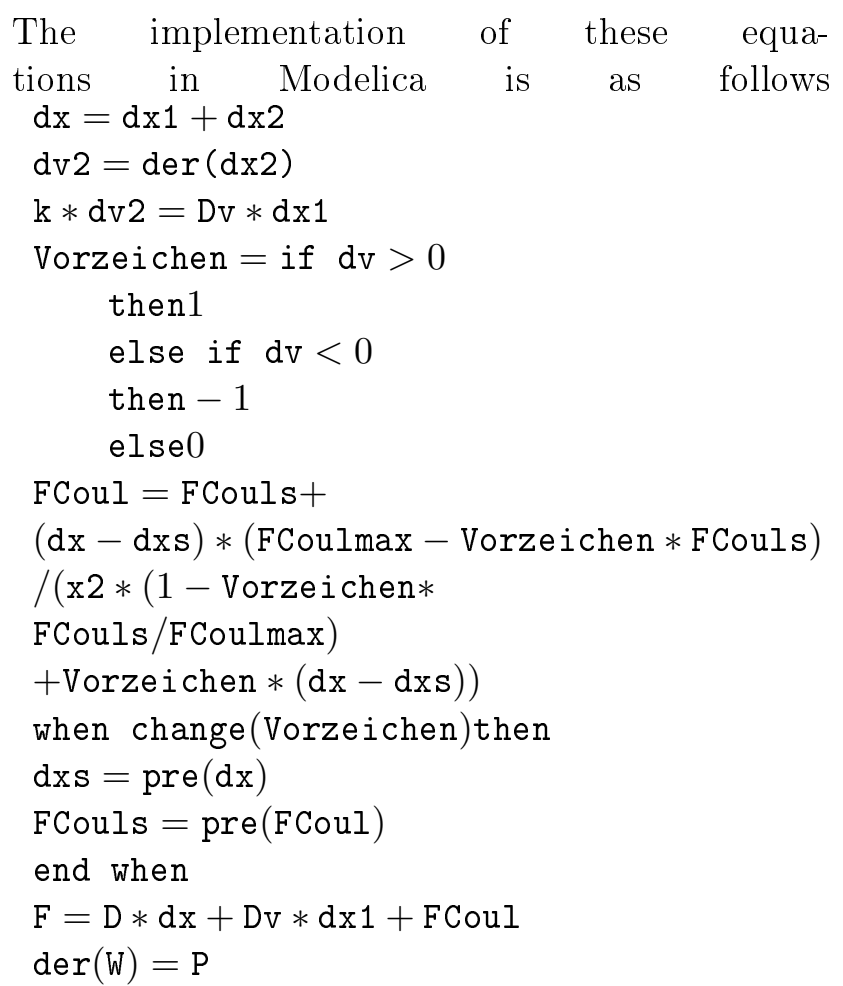

The implementation of these equations in Modelica is as follows $\mathrm{dx}=\mathrm{dx} 1+\mathrm{dx} 2$

$\mathrm{dv} 2=\operatorname{der}(\mathrm{dx} 2)$

$\mathrm{k} * \mathrm{dv} 2=\mathrm{Dv} * \mathrm{dx} 1$

Vorzeichen $=$ if $\mathrm{dv}>0$

then 1

else if $\mathrm{dv}<0$

then -1

else 0

FCoul $=$ FCouls +

$(\mathrm{dx}-\mathrm{dxs}) *($ FCoulmax - Vorzeichen $*$ FCouls $)$

$/(\mathrm{x} 2 *(1-$ Vorzeichen $*$

FCouls/FCoulmax)

+ Vorzeichen $*(\mathrm{dx}-\mathrm{dxs}))$

when change(Vorzeichen)then

$\mathrm{dxs}=\operatorname{pre}(\mathrm{dx})$

FCouls $=\operatorname{pre}($ FCoul $)$

end when

$\mathrm{F}=\mathrm{D} * \mathrm{dx}+\mathrm{Dv} * \mathrm{dx} 1+\mathrm{FCoul}$

$\operatorname{der}(\mathrm{W})=\mathrm{P}$ 
where $\mathrm{dx} 1$ is the displacement of spring Dv and $\mathrm{dx} 2$ is the displacement of the damper $k$.

To illustrate the behavior of the M. Berg friction model, it (Figure 5) is compared to a diode model for Coulomb friction, similar to the one of the Modelica Standard Library (Figure 6), as described for example in reference [7]. As can be seen from these graphs, the hysteresis loop of the Modelica Standard Library model has a rectangular form, whereas the M. Berg friction model shows sharp corners at the maximum and minimum displacements with a smooth transition between the upper and lower branch of the hysteresis.

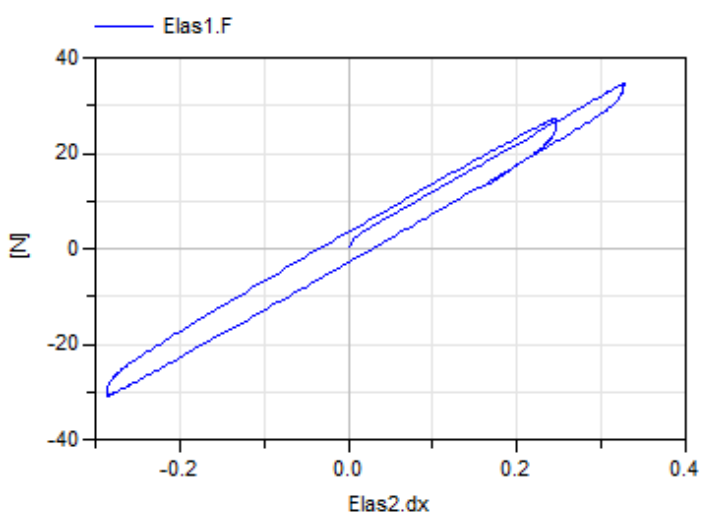

Figure 5: Friction force versus displacement. Simulation of the friction model for a rubber spring proposed by M.Berg. $F_{\max }=2$ and $x_{2}=0.002$.

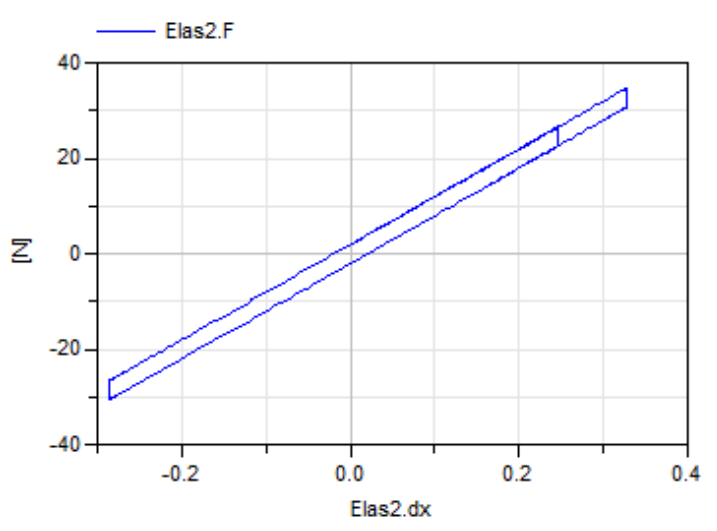

Figure 6: Friction force versus displacement. Simulation of the friction model for the basic model for Coulomb friction in the Modelica Standard Library. $F_{\max }=2$ and $x_{2}=0.002$.

In the final model for the Miner buffer in the DyMoRail library, the total force:

$$
F=F_{e}+F_{v}+F_{C o u l}
$$

is taken to the exponent of an exponential function. This corresponds more to reality, shown by a thorough parameter study.

\section{Simulation}

For their acceptance test, railway companies define several crash scenarios with different crash partners at different speeds.

Figure 7 shows simulations for 40 J-buffer known as "Miner40" at different initial speeds. This type of buffers is exclusively used for freight wagons. The acceptance tests demand reversible shunting at speeds up to $12 \mathrm{~km} / \mathrm{h}$. During collisions at low speeds, the energy has to be absorbed by the buffers. Absolutely no damage should occur to the rolling stock. The acceleration must remain below $4.0 \mathrm{~g}$.

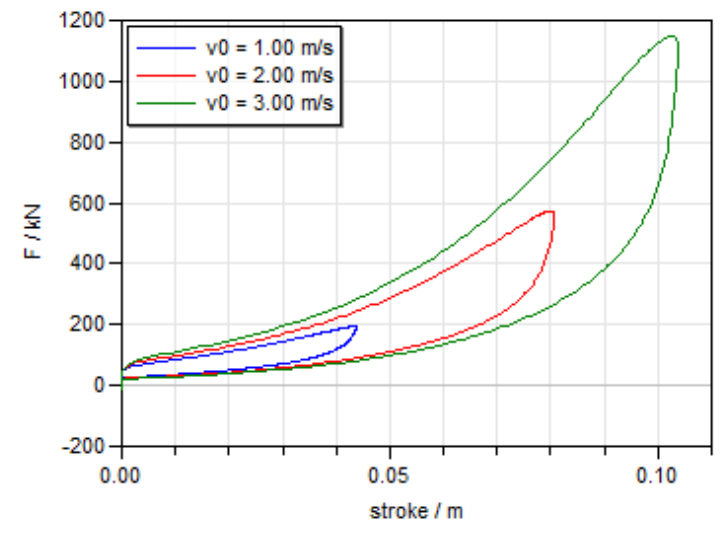

Figure 7: Simulation of the buffer model with different initial speeds. Force vs stroke diagram.

The elastomer model works also in combination with other coupler or buffer models in complete train configuration. Figure 8 shows a simulation of three S-Bahn combinations colliding with a single combination at rest during shunting. Each S-Bahn contains four cars and three Miner models, so in total the model contains sixteen cars and fifteen buffer models. In this graph, one can distinguish the different cars colliding one after the other.

\section{Conclusion}

In this paper a model for elastomer buffers for railway vehicle dynamics is presented. This model is part of the more extended DyMoRail library which allows to simulate longitudinal dynamics of entire 


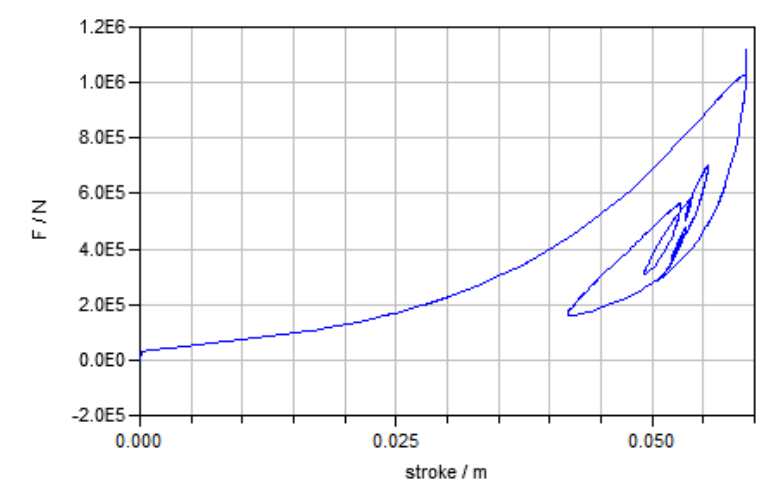

Figure 8: Simulation of three S-Bahn combinations colliding with a single combination at rest during shunting.

railway trains. With this library an efficient simulation of complete train compositions in various combinations is possible. The elastomer buffer can be used in combination with other buffer and couplers models. The acceptance test scenarios can be simulated with the help of this model.

\section{Acknowledgements}

This project has been funded by CTI (Swiss Federal Commission for Technology and Innovation, contract number 14165.1).

\section{References}

[1] Berg, M. A model for rubber springs in the dynamic analysis of rail vehicles, Proc Instn Mech Engrs, Vol 211 Part F (1997), pp. 95108.

[2] Berg, M. A Non-Linear Rubber Spring Model for Vehicle Dynamics Analysis, Vehicle System Dynamics Supplement, Swets and Zeitlinger Publishers, 28 (1998), pp. 723-728.

[3] Berg, M. A Non-Linear Rubber Spring Model for Rail Vehicle Dynamics Analysis, Vehicle System Dynamics, Swets and Zeitlinger Publishers, 30 (1998), pp 197 - 212.

[4] Dumont E. and Maurer W. Proceedings of the 9th International Modelica Conference, 2012, p.691-696.

[5] Maurer W. Puffer nach Mass. Eisenbahn Revue 3,2003 , p.118-119.
[6] Maurer W. Simulationsgestütze Entwicklung von Puffern und Dämpfern für Eisenbahnzüge. Proceedings of the 18th Symposium on Simulationtechnique ASIM 2005, Erlangen, Germany, ASIM September 12-15 2005 .

[7] Otter M., Elmqvist H., Mattsson S.-E. Hybrid Modeling in Modelica based on the Synchronous Data Flow Principle, Proceedings of the 1999 IEEE Symposium on Computer Aided Control System Design (CACSD'99), Hawaii, Aug. 22-27, 1999. 